DOI: $10.3901 / J M E .2019 .14 .019$

\title{
位感条纹三维振动测量原理及试验研究"
}

\author{
钟剑锋 ${ }^{1}$ 钟舜聪 $^{1}$ 彭志科 $^{2}$ \\ (1. 福州大学机械工程及自动化学院 福州 350116; \\ 2. 上海交通大学机械系统与振动国家重点实验室 上海 200240)
}

\begin{abstract}
摘要: 针对当前单目视觉测量方法难以实现结构三维振动同步测量的问题, 提出一种基于位感条纹和高速相机的结构三维振 动同步测量新方法。在建立测量系统完整的原理模型基础上，系统描述单相机成像坐标下采用单个位感条纹获取结构三维动 态位移信息的测量原理, 建立成像位感条纹三维编码动态参数与结构实际三维位移之间的内在联系, 推导各维位移的计算公 式。通过结构三维运动和视觉成像系统的联合仿真模型, 验证位移测量原理和算法的正确性; 并通过仿真和试验分析系统的 测量性能及其影响因素。通过对结构已知三维运动的测量, 并与基于三维加速度传感器的接触式测量方法进行对比试验, 验 证基于位感条纹的视觉测量方法及系统在实现结构三维振动位移准确同步测量的有效性。
\end{abstract}

关键词: 位感条纹; 高速相机; 三维振动; 同步测量

中图分类号: TG156

\section{Theoretical and Experimental Studies on 3D Vibration Measurement Based on Displacement-sensitive Fringe Pattern}

\section{ZHONG Jianfeng ${ }^{1}$ ZHONG Shuncong ${ }^{1} \quad$ PENG Zhike $^{2}$}

(1. School of Mechanical Engineering and Automation, Fuzhou University, Fuzhou 350116;

2. State Key Laboratory of Mechanical System and Vibration, Shanghai Jiao Tong University, Shanghai 200240)

\begin{abstract}
A novel method for the measurement of structural three-dimensional (3D) vibration based on a displacement-sensitive fringe pattern (DSFP) and a high speed camera is proposed to overcome the simultaneous measurement problem in structural 3D vibration using monocular vision. Based on the established model of the proposed monocular vision measurement system, the measurement principle for structural 3D vibration is illustrated systematically. The relationships between the real 3D displacement and the 3D dynamics parameters obtained from the recorded DSFP image sequence is established. The formulas for calculating each dimensional displacement is also derived. The correctness of the displacement measurement principle and algorithm is verified by using the co-simulation model of structural 3D motion and monocular vision system. The performance and its influencing factors of the measurement system are evaluated by simulation and experiment. The experimental results for known structural 3D displacement and the contrast test results with the 3D acceleration sensor demonstrates the effectiveness of the proposed DSFP and vision based system in structural 3D displacement measurement.
\end{abstract}

Key words: displacement-sensitive fringe pattern; high-speed camera; 3D vibration; simultaneous measurement

\section{0 前言}

振动信号可以为结构的安全评估和健康监测提 供丰富的有效信息。振动测量技术按其与测量对象 接触与否可以分为接触式测量和非接触式测量。接 触式振动测量传感器, 如加速度传感器、接触式位

* 国家自然科学基金(51675103)、机械系统与振动国家重点实验室开放课题 基金(MSV-2018-07)、上海市自然科学基金(18ZR1414200)和福建省科技计 划(201910004)资助项目。20180503 收到初稿, 20190327 收到修改稿
移传感器等，在一些场合会因其与结构的物理连接 或自身附加质量对测量结构的振动特性产生一定的 影响而受到限制。非接触式测量方法可实现非侵入 式的测量, 结构动态特性不会受到测量传感器附加 质量或外力的影响, 可用于结构模态参数的准确测 量, 特别适用于一些轻质结构的测量。

机器视觉测量技术作为一种新的测量技术, 其 以非接触、高效率、高精度、无负载效应等特点受 到越来越多研究者的关注 ${ }^{[1]}$ 。并且, 随着数字成像 技术与图像处理技术的发展, 二维图像传感器在成 
像分辨率和成像速度方面都有较大提升, 促进了视 觉测量技术的快速发展。该类方法先采用相机对振 动结构表面的标靶图案或自然特征进行连续采集成 像, 再采用不同图像处理技术实现被测结构振动、 形变、位移等物理参数的测量。投影条纹法 ${ }^{[2]}$ 是一 种基于机器视觉的非接触式全场光学测量方法, 该 方法通过采集投影在物体表面的条纹实现其形貌或 振动的测量。 $\mathrm{TAY}^{[3]}$ 采用投影条纹和机器视觉实现 微小构件的动态测量。MITCHELL ${ }^{[4]}$ 采用条纹投影 和机器视觉测量技术实现结构的模态分析。陈露 等 ${ }^{[5]}$ 采用条纹投影实现扬声器的形变测量。该类方 法通过采集的投影条纹强度信号获得条纹的相位信 息, 测量精度容易受到光强波动的影响。并且需要 相位解包裹算法 ${ }^{[3]}$ 来对不连续相位进行解包裹以获 得连续变化的相位信息。另外, 该方法在测量时需 要确定的系统参数较多, 给测量带来一些不便。

也有研究者采用图像对比跟踪算法 ${ }^{[6]}$ 、模板匹 配技术 ${ }^{[7]}$ 和边缘检测技术 ${ }^{[8]}$ 等实现结构的动态测量。 DOIGNON 等 ${ }^{[9]}$ 等采用双目视觉测量系统, 结合边 缘检测算法和深度计算算法实现卷绕传输系统中移 动卷绕物的横向位移及离面振动的测量。BUSCA 等 ${ }^{[7]}$ 采用模板匹配、边缘检测算法对安装于铁路桥 表面的不同目标图像在图像传感器中的位置变化进 行探测, 实现了桥梁垂直位移的测量。FUKUDA 等 ${ }^{[6]}$ 和 FENG 等 ${ }^{[10]}$ 结合机器视觉测量、目标匹配和 方向码匹配算法捕捉结构自然特征的运动实现结构 的位移测量。KHUC 等 ${ }^{[11]}$ 也采用普通相机实现结构 自然特征的运动检测与匹配, 在不需要标靶图案的 情况下实现结构的振动测量。国内学者季云峰 ${ }^{[12]}$ 采 用长焦镜头的普通相机和无目标计算机视觉技术实 现拉索桥拉索的振动测量与分析。

数字图像相关法是视觉测量提取结构动态信息 的一种常见算法, 可分为二维数字图像相关法 ${ }^{[13-15]}$ 和三维数字图像相关法 ${ }^{[16]}$ 。二维图像相关技术只需 一个二维图像传感器, 适用于面内位移的测量; 三 维数字图像相关技术则需要两个二维图像传感器对 图像进行同步采集以实现结构三维振动的测量。数 字图像相关法优点在于装置简单, 具有较高的测量 灵敏度和分辨率 ${ }^{[13]}$ 。陈忠等 ${ }^{[17]}$ 采用双目立体视觉实 现结构全场振动的测量。歹英杰等 ${ }^{[18]}$ 采用双目立体 机器视觉测量空间目标的三维运动参数及空间姿态 等。上述测量技术在采用单个相机时一般只能实现 平行于成像传感器平面结构二维动态位移的测量, 要实现三维信息测量则需要采用两个相机进行同步 采集。

近年来，作者提出两种基于白光干涉条纹的一
维 ${ }^{[19]}$ 和二维 ${ }^{[20]}$ 光学相干振动层析系统, 该类系统可 实现结构亚纳米级精度的单点或多点一维振动的测 量, 但该类测量方法需要搭建精密的干涉光路才能 实现。结合基于干涉条纹的二维光学相干振动层析 系统和基于机器视觉的非干涉振动测量技术各自的 优点，作者提出一种基于类干涉条纹和高速相机的 测量系统。该系统采用类似于干涉条纹的图片粘贴 于结构表面作为振动传感器, 并采用高速相机为探 测器实现结构的振动测量 ${ }^{[21]}$ 、模态分析 ${ }^{[22]}$ 和缺陷检 测 ${ }^{[23]}$ 。该测量方法主要可实现结构沿成像光轴方向 微米量级到厘米量级振动位移的测量。本文在前期 研究的基础上, 针对单目视觉测量系统只能实现两 个维度振动测量的不足, 设计了一种新型位感条纹 作为三维位移传感器, 以高速相机作为探测器即可 实现结构三维振动位移的同步测量。与其他研究者 提出的基于单个相机的测量系统相比，扩展了单相 机测量系统的测量维度、提高了测量效率。

\section{1 系统组成及原理}

\section{1 系统组成}

基于位感条纹和高速相机的新型三维振动测量 系统原理如图 1 所示。该测量系统主要由位感条纹、 高速相机和电脑组成。位感条纹作为三维振动传感 器附于被测结构表面; 高速相机用于结构振动过程 中位感条纹图像的采集; 电脑用于高速相机的控制 及采集位感条纹图像的处理。该振动测量系统的三 维测量坐标定义如图 1 所示: 将沿成像光轴方向定 义为 $X$ 轴, 坚直方向定义为 $Y$ 轴, 水平方向定义为 $Z$ 轴。

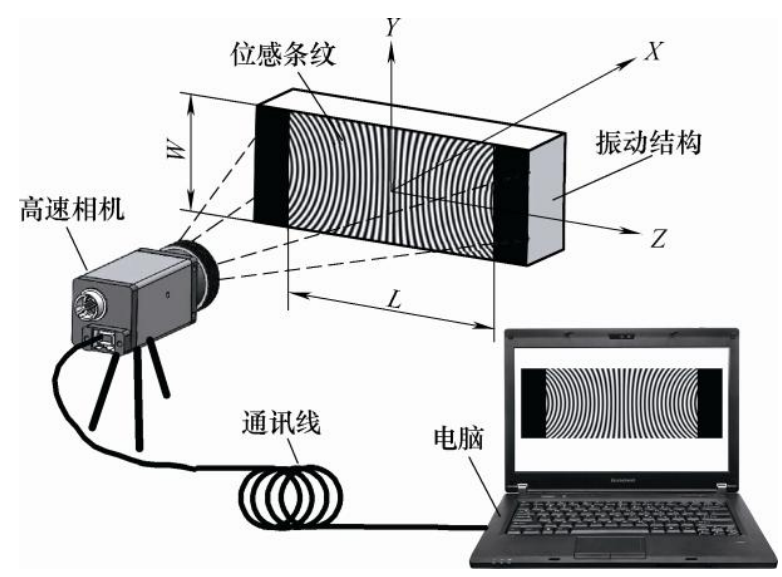

图 1 基于位感条纹和高速相机的三维振动测量系统

位感条纹形式如图 1 所示, 该位感条纹通过 Matlab 软件编程产生, 再将其保存成图片并采用普 通打印机将其打印到背胶相片纸上，即完成位感条 纹传感器的制作。位感条纹有效条纹长度和宽度分 
别为 $L$ 和 $W$, 其值可由测量结构的尺寸和成像距离 决定。该位感条纹的特点在于沿长度方向的条纹密 度固定, 而沿宽度方向的条纹密度按二次函数变化。 因此, 该位感条纹中心线位置对应于该二次变密度 曲线斜率值为零的位置。在被测结构表面粘贴好位 感条纹后, 应使位感条纹图像成像于图像传感器的 中心位置, 并调整成像镜头的参数使位感条纹清晰 成像。

\section{$1.2 X$ 方向位移测量原理}

被测结构沿成像光轴方向的位移 $\Delta x(t)$ 可由成 像位感条纹宽度方向中心线位置的条纹密度变化获 得, 其测量原理如图 2 所示, 图 2a、2b 分别为测量 系统侧视图和俯视图。因为制作完成的位感条纹有 效条纹宽度和长度是固定的, 所以图像传感器中位 感条纹图像的成像宽度和长度将随着 $X$ 方向位移的 变化而变化, 从而使位感条纹图像宽度方向中心线 处的条纹密度发生变化。假设 $t$ 时刻结构在 $X$ 方向 的位移为 $\Delta x(t)$, 根据文献[23]可得沿 $X$ 方向的位移 可表达为

$$
\Delta x(t)=\frac{D\left(d(t)-d_{r}\right)}{d_{r}}
$$

式中, $D$ 为成像系统的成像物距, $d_{r}$ 和 $d(t)$ 分别为 参考位感条纹中心线位置条纹的条纹密度和结构在 成像光轴 $X$ 方向有 $\Delta x(t)$ 位移时位感条纹中心线位 置条纹的条纹密度。

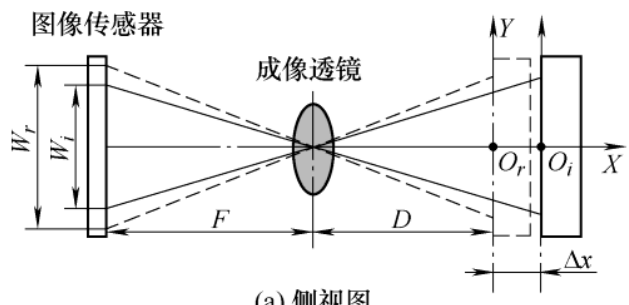

(a) 侧视图

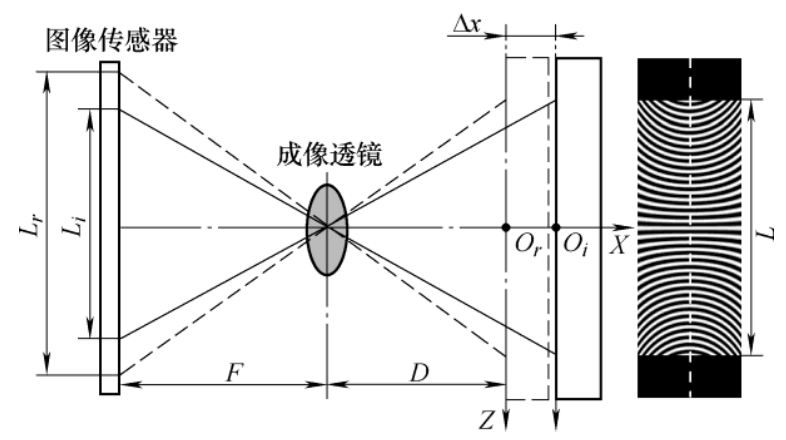

(b) 俯视图

图 2 系统 $X$ 方向位移测量原理

\section{$1.3 Y$ 方向位移测量原理}

结构 $Y$ 方向的位移测量原理如图 3 所示, 图 3a 中结构只沿 $Y$ 方向有位移, 该位移可以通过求取位 感条纹宽度方向中心线在图像传感器中的位移 $h$ 获
得。由图中成像关系可得, 结构在 $t$ 时刻只沿 $Y$ 方 向有位移 $\Delta y(t)$ 时, 该位移可表达为

$$
\Delta y(t)=D \cdot \tan (\alpha(t))=D \frac{h(t)}{F}=D \frac{a \Delta m(t)}{F}
$$

式中, $F$ 为图像传感器和透镜之间的成像距离, $h(t)$ 为位感条纹中心线在图像传感器上相对于参考条纹 中心线的位移, 该位移可由等式 $h(t)=a \Delta m(t)$ 计算 获得, 其中 $a$ 为图像传感器的像素点宽度, $\Delta m(t)$ 为 $t$ 时刻位感条纹中心线在图像传感器上相对于参考 条纹中心线偏移的像素点数。

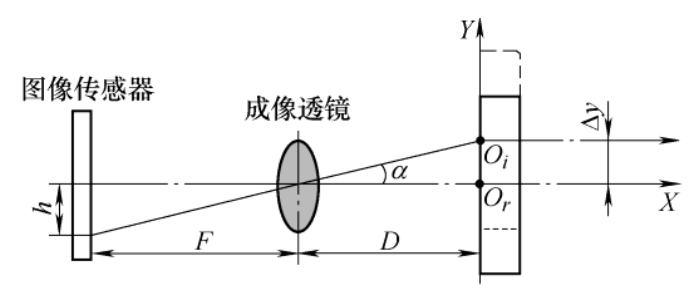

(a) 结构只沿 $Y$ 方向有位移

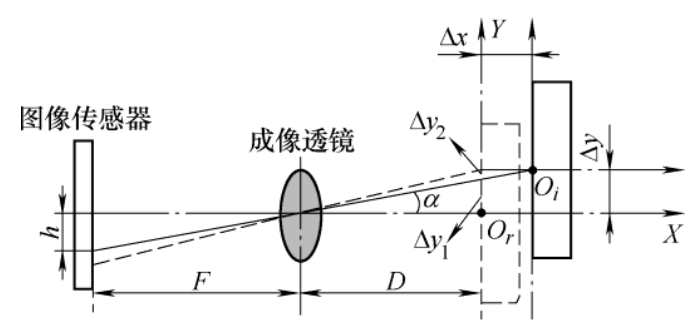

(b) 结构沿 $X$ 和 $Y$ 方向都有位移

图 3 系统 $Y$ 方向位移测量原理

由图 3b 可知, 当结构沿 $X$ 和 $Y$ 轴方向同时有 位移时, $X$ 方向位移将影响 $Y$ 方向位移的计算。 考虑 $X$ 方向位移的影响, 沿 $Y$ 轴方向的位移可表 达为

$$
\begin{gathered}
\Delta y(t)=\Delta y_{1}(t)+\Delta y_{2}(t)= \\
D \cdot \tan (\alpha(t))+\Delta x(t) \cdot \tan (\alpha(t))
\end{gathered}
$$

根据式(2)可得, 在 $t$ 时刻 $\tan (\alpha(t))$ 的值可表达为

$$
\tan (\alpha(t))=\frac{h(t)}{F}=\frac{a \Delta m(t)}{F}
$$

将式(1)、(4)代入式(3)化简可得到 $Y$ 轴方向位移 表达式为

$$
\Delta y(t)=\frac{D a \Delta m(t) d(t)}{d_{r} F}
$$

又因为该成像系统的成像比例可由 $D$ 和 $F$ 之间 的比值获得，即

$$
M_{a}=\frac{D}{F}=\frac{L}{a N_{L}}
$$

式中, $L$ 为位感条纹有效条纹的实际长度, $N_{L}$ 为位 感条纹有效条纹长度在图像传感器中覆盖的像素点 数。将式(6)代入式(5)化简可得 


$$
\Delta y(t)=\frac{L \Delta m(t) d(t)}{d_{r} N_{L}}
$$

由式(1)、(7)可知, 获得结构在 $X$ 和 $Y$ 方向位移 $\Delta x(t)$ 和 $\Delta y(t)$ 的关键在于准确确定位感条纹中心线 在图像传感器中的坐标位置。只有得到位感条纹的 中心位置后才能准确得到该中心位置的条纹密度值 $d(t)$ 和 $Y$ 方向的偏移值 $h(t)$ 。在实际测量系统中, 得到的条纹密度变化曲线采样分辨率受到图像传感 器分辨率的限制, 在求取条纹密度变化曲线斜率为 零坐标点时可能存在较大的误差。因此在获得条纹 密度变化曲线后可先对曲线进行拟合平滑, 再求其 斜率曲线, 最后通过插值法求得条纹密度变化曲线 斜率为零的坐标位置, 实现结构 $Y$ 方向位移的精确 测量。

\section{$1.4 Z$ 方向位移测量原理}

结构 $X$ 和 $Y$ 方向的位移分别可以从位感条纹宽 度方向中心线处的条纹密度变化和位感条纹中心线 在图像传感器中纵坐标位置的变化获得。同理, 结 构在 $Z$ 方向的位移可以通过位感条纹有效条纹长度 方向的中心点在图像传感器中的坐标位置变化求 得，其测量原理如图 4a 所示。

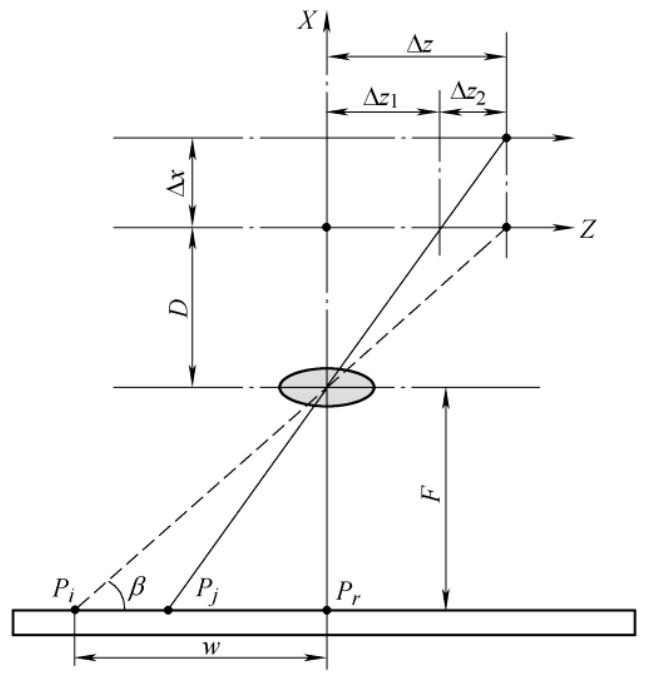

(a) 俯视图

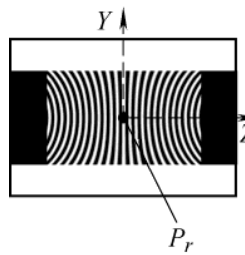

(b) 参考条纹图

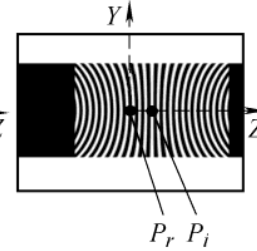

(c) 第 $i$ 帧条纹图

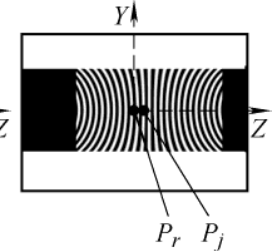

(d) 第 $j$ 帧条纹图
图 4 系统 $Z$ 方向位移测量原理

假设粘贴于结构表面的位感条纹在图像传感器 中的初始成像位置位于图像传感器的中心, 即其参 考中心点 $P_{r}$ 位于图像传感器的中心, 如图 $4 \mathrm{~b}$ 所示。 在 $t$ 时刻结构只在 $Z$ 方向有位移 $\Delta z(t)$ 时, 位感条纹
在图像传感器中的图像及其位置如图 4c 所示, 此时 位感条纹长度方向的中心点 $P_{i}$ 与参考中心点 $P_{r}$ 存 在一定位移。根据图 $4 \mathrm{a}$ 中的三角成像关系可得

$$
\tan (\beta(t))=\frac{F}{w(t)}=\frac{D}{\Delta z(t)}
$$

即

$$
\Delta z(t)=\frac{D w(t)}{F}
$$

将式(6)代入式(9)可得

$$
\Delta z(t)=\frac{L w(t)}{a N_{L}}=\frac{L a \Delta n(t)}{a N_{L}}=\frac{L \Delta n(t)}{N_{L}}
$$

式中, $w(t)$ 为位感条纹长度方向的中心点在 $t$ 时刻 偏移图像传感器参考中心 $P_{r}$ 的距离, 该距离可由等 式 $w(t)=a \Delta n(t)$ 计算获得, 其中 $\Delta n(t)$ 为位感条纹长 度方向的中心点在图像传感器上沿 $Z$ 方向偏移图像 传感器中心 $P_{r}$ 的像素点数。

当结构在 $X$ 和 $Z$ 方向同时有位移时, 即当位感 条纹的中心点位于图 $4 \mathrm{a}$ 中的 $P_{j}$ 位置时, 结构在 $X$ 方向位移将对成像位感条纹长度方向的中心点位置 有影响, 如图 $4 \mathrm{~d}$ 所示。考虑 $X$ 方向位移的影响, 结构在 $Z$ 方向的位移为

$$
\begin{gathered}
\Delta z(t)=\Delta z_{1}(t)+\Delta z_{2}(t)= \\
\frac{D}{\tan (\beta(t))}+\frac{\Delta x(t)}{\tan (\beta(t))}
\end{gathered}
$$

将式(1)和(8)代入并化简可得

$$
\Delta z(t)=\frac{D d(t)}{d_{r} \tan (\beta(t))}=\frac{D d(t) w(t)}{d_{r} F}
$$

再将式(6)代入式(12)化简可得

$$
\Delta z(t)=\frac{L a \Delta n(t) d(t)}{a N_{L} d_{r}}=\frac{L \Delta n(t) d(t)}{N_{L} d_{r}}
$$

通过式(13)即可消除 $X$ 方向位移对 $Z$ 方向位 移的影响。

因此，要获得结构在 $Z$ 方向的位移，必须获得 位感条纹有效条纹长度方向的中心点在图像传感器 上沿 $Z$ 方向偏移图像传感器参考中心 $P_{r}$ 的准确距 离。如图 $4 \mathrm{c}$ 所示, 当结构只在 $Z$ 方向有位移时, 位 感条纹宽度方向中心线处的条纹密度保持不变。此 时，位感条纹宽度方向中心线处的有效条纹在图像 传感器中的像素偏移量可以通过其与参考位感条纹 中心线处条纹信号相关序列的最大峰值点坐标确 定。但是, 当结构在 $X$ 和 $Z$ 方向有位移时, 成像位 感条纹中心线处的条纹密度将发生变化, 即有效条 纹的空间频率将发生变化, 如果采用参考位感条纹 中心线处条纹信号与密度变化后的条纹进行相关运 
算时, 其相关系数将随着两条纹信号频率差的增大 而减小。若因为 $X$ 方向位移过大导致两条纹信号的 频率差过大, 它们之间将不相关, 也就无法通过搜 索互相关序列峰值坐标位置确定有效条纹在 $Z$ 方向 的像素偏移量。

针对该问题, 可以通过当前条纹密度的变化重 新生成与该条纹密度相同的且位于图像传感器中心 的条纹信号作为参考条纹。保证用于相关运算的条 纹信号频率相等, 使相关序列峰值位置坐标永远存 在。假设图像传感器宽度方向总的成像像素点为 $N_{a}$, 初始帧位感条纹中心线处的有效条纹周期数和 覆盖的像素点分别为 $p$ 和 $N_{L}$, 则在 $t$ 时刻位感条纹 中心线条纹覆盖的像素点为

$$
N(t)=\frac{N_{L} d_{r}}{d(t)}
$$

在 $t$ 时刻生成的位于图像传感器中心位置的参 考条纹在图像传感器中的分布函数为

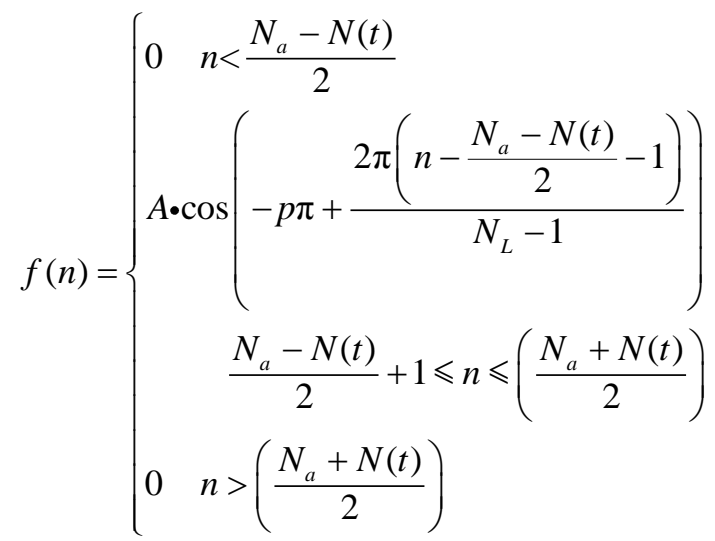

式中, $n$ 为图像传感器的横向坐标, $A$ 为生成条纹 的强度幅值, 其值与采集得到的位感条纹中心线处 的条纹强度幅值相等。通过式(15)即可求得与 $t$ 时刻 中心线条纹周期密度相等且位于图像传感器中心位 置的参考条纹信号。再将 $t$ 时刻的移位条纹信号与 生成的参考条纹信号进行互相关运算, 得出相关序 列峰值坐标即可确定位感条纹长度方向的中心偏离 图像传感器参考中心位置的像素点数 $\Delta n(t)$ 。最后根 据式(13)得到 $Z$ 方向的位移信号。在实际的测量过 程中, 有效条纹的采样分辨率也受到图像传感器分 辨率的限制, 在采用互相关运算确定峰值坐标位置 时其坐标分辨率与像素点间距相同。为了提高系统 在 $Z$ 方向的位移分辨率, 在进行互相关运算之前可 先对两条纹信号进行插值处理。实际的插值点数可 根据位感条纹图像实际的分辨率和希望得到的位移 分辨率进行选择, 一般该位移分辨率会随着插值点 数的增加而提高。

\section{5 位感条纹中心线的确定}

由上述分析可知, 采用该系统实现结构三维振
动测量的关键在于精确确定位感条纹宽度方向的中 心线位置。如图 5a 中虚线所示, 该中心位置与条纹 密度变化曲线斜率为零的坐标位置相等。因此, 要 精确获得该中心位置必须获得准确的条纹密度变化 曲线, 该密度变化曲线可以通过求每行条纹的频率 获得, 通常可采用快速傅里叶变换求每行条纹的频 率。但是因为该位感条纹的长度是有限的, 采用快 速傅立叶变换计算时会因为频谱泄露而不能获得条 纹精确的密度信息。为了获得更为精确的条纹频率 信息, 在对每行条纹频率进行计算时采用能量重心 校正法 ${ }^{[24]}$ 进行校正, 以得到更为准确的条纹密度变 化曲线。

在进行频谱较正时先对采集的干涉条纹信号加 量纲一化的 Hanning 窗函数以减小其频谱泄露, 该 窗函数可表达为

$W(m)=0.5-0.5 \cos \left(\frac{2 \pi m}{N_{a}}\right) \quad m=0, \cdots, N_{a}-1$

式中, $N_{a}$ 为采集得到的位感条纹沿长度方向总采样 点数。当式(16)运用到采集的条纹信号时, 其量纲 一化的频谱主瓣模函数的平方为

$$
G_{\omega^{\prime}}=\frac{A^{2} \sin ^{2}\left[\pi\left(\omega^{1}-\omega_{i}^{1}\right)\right]}{4 \pi^{2}\left(\omega^{1}-\omega_{i}^{1}\right)^{2}\left[1-\left(\omega^{1}-\omega_{i}^{1}\right)^{2}\right]^{2}}
$$

式中, $\omega_{i}^{1}$ 和 $A$ 分别为分析的第 $i$ 行条纹信号的量纲 一化频率和幅值。根据所加量纲一化 Hanning 窗函 数的能量重心分布特性可以得出

$$
\begin{gathered}
\sum_{j=-z}^{z} G_{k+j}\left(k-\omega_{i}^{1}+j\right)= \\
\sum_{j=-z}^{z}(k+j) G_{k+j}-\omega_{i}^{1} \sum_{j=-z}^{z} G_{k+j}
\end{gathered}
$$

式中, $G_{j}$ 为第 $j$ 条谱线幅值, $G_{k}$ 为主瓣内最大的谱 线幅值, $k$ 为最大谱线幅值对应的谱线号。那么第 $i$ 行条纹的主瓣中心量纲一化频率

$$
\omega_{i}^{1}=\frac{\sum_{j=-z}^{z}(k+j) G_{k+j}}{\sum_{j=-z}^{z} G_{k+j}}
$$

经能量重心频谱校正法校正后可得到条纹沿 宽度方向的精确的频率变化曲线(即条纹密度变 化曲线), 如图 5b 所示。得到条纹的密度变化曲 线后对其进行多项式拟合平滑，并求得平滑后的 密度变化曲线的斜率, 通过插值求出斜率为零的 坐标, 即可确定位感条纹在宽度方向的中心线位 置, 如图 5c 所示。 


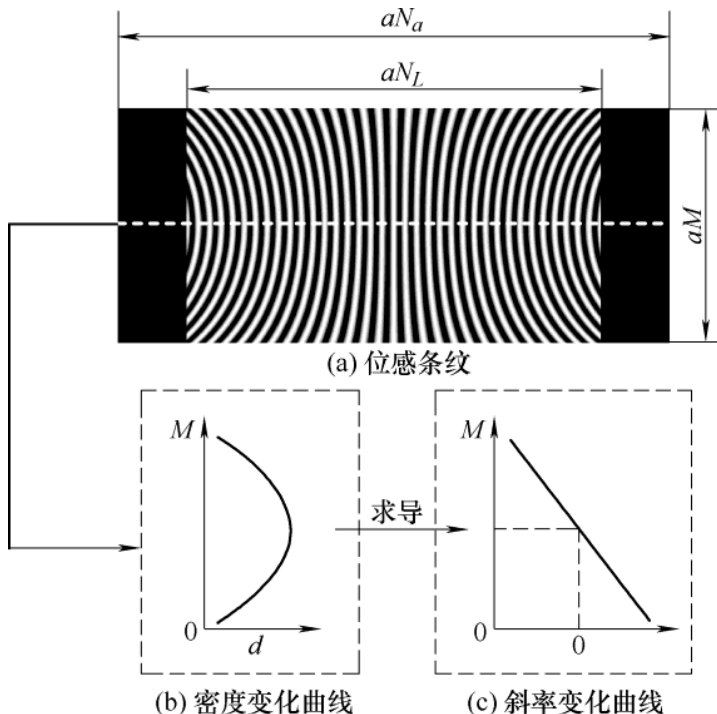

图 5 位感条纹图及宽度方向中心线确定原理

\section{2 系统测量性能影响因素分析}

由系统三维位移测量原理可知, 该系统主要采 用高速相机对位感条纹进行成像以实现结构的三维 振动测量。虽然采用能量重心频谱校正法可实现条 纹密度信息的精确提取, 但是该算法的精度会受到 位感条纹图像分辨率和信噪比的影响。而位感条纹 质量主要由位感条纹的打印质量和成像系统的成像 质量决定。位感条纹的打印质量可以通过增加位感 条纹的分辨率和采用打印质量高的打印机进行打印 得到保证。而成像系统的成像质量却受到诸多因素 的影响, 如环境光照、成像镜头、图像传感器分辨 率、图像位深等。通常相机图像传感器分辨率是固 定的, 为了获得更好的图像分辨率应尽量使更多的 像素点用于位感条纹的成像。所以在实际的测量过 程中应该根据成像距离选择合适的成像镜头。另外, 由于外界环境光照条件、图像采样位深和成像噪声 等因素的影响, 成像位感条纹的图像信噪比也各不 相同。

为了讨论位感条纹图像分辨率和信噪比对系统 三维位移测量性能的影响规律, 采用 SOLIDWORKS 软件的运动仿真模块建立结构三维 振动模型, 在结构的表面设置同样的位感条纹, 并 采用软件的成像模块对该位感条纹进行成像。为了 评估该系统三维位移测量的不确定度与位感条纹图 像分辨率和信噪比的关系, 对静止结构表面的位感 条纹进行拍摄。模拟中采集了两组分辨率分别为 $476 \times 238$ 像素点和 $1276 \times 638$ 像素点的位感条纹 序列, 每组序列为 200 张。然后对不同分辨率的两 组位感条纹序列图像加入不同量级的高斯白噪声,
分别生成九组条纹信噪比分别为 10、20、30、40、 50、60、70、80 和 $90 \mathrm{~dB}$ 的条纹序列。然后采用提 出的位移算法计算各组条纹序列的三维位移并计算 位移的标准偏差来评估位移测量的不确定度, 模拟 结果如图 6 所示。由图可知, 当位感条纹图的分辨 率固定时, 其各方向的位移不确定度随着条纹图像 信噪比的增加而减小。从图 $6 \mathrm{a}$ 和 $6 \mathrm{~b}$ 对比可以看出, 增加位感条纹的图像分辨率也可以减小各方向位移 的测量不确定度。从不同方向的位移不确定度变化 曲线可以发现, 在条纹图像信噪比低于 $50 \mathrm{~dB}$ 时, 该测量系统 $X$ 方向的位移不确定度比 $Y$ 和 $Z$ 方向的 不确定度要大得多。而随着条纹质量的提高, 测量 系统三个方向的位移不确定度差别越来越小。当位 感条纹分辨率为 $1276 \times 638$ 像素点、信噪比为 $50 \mathrm{~dB}$ 时, $X 、 Y$ 和 $Z$ 方向的位移不确定度分别为 $0.1 \mu \mathrm{m}$ 、 $0.2 \mu \mathrm{m}$ 和 $0 \mu \mathrm{m}$ 。因此, 在获得高质量的位感条纹图 像的情况下, 该系统可获得较好的三维位移分辨率。

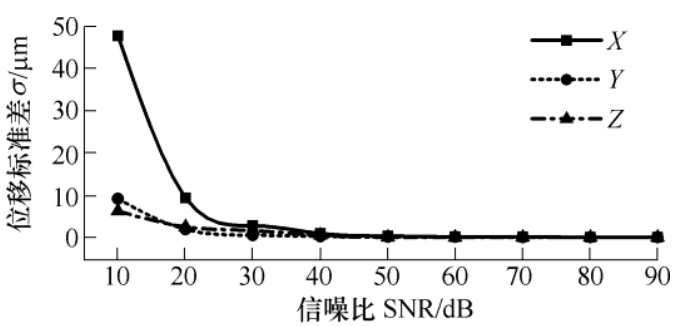

(a) 位感条纹成像分辨率为 $476 \times 238$ 像素点

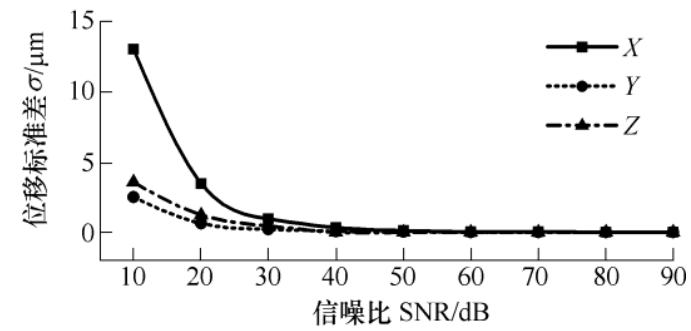

(b) 位感条纹成像分辨率为 $1276 \times 638$ 像素点

图 6 位感条纹图分辨率和信噪比与位移不确定度的关系

\section{3 试验及分析}

为了验证提出的基于位感条纹的结构三维振动 测量系统的可行性与准确性。搭建了一套试验测量 系统分别对安装于三维电动移动平台的轻质平板结 构和安装于激振器输出端悬臂梁的三维振动进行测 量, 试验装置如图 7 所示。试验中采用型号为 PCO. $1200 \mathrm{hs}$ 的 CMOS 高速相机, 其最大分辨率为 $1280 \times 1024$ 像素, 全分辨率下的最高帧率为 636 帧/s, 单个像素点尺寸和位深分别为 $12 \mu \mathrm{m} \times 12 \mu \mathrm{m}$ 和 8 位。 成像系统使用的成像镜头型号为 Nikon 24-85 mm F2.8-4D。位感条纹有效条纹长度和宽度分别为 40 
$\mathrm{mm}$ 和 $20 \mathrm{~mm}$ 。

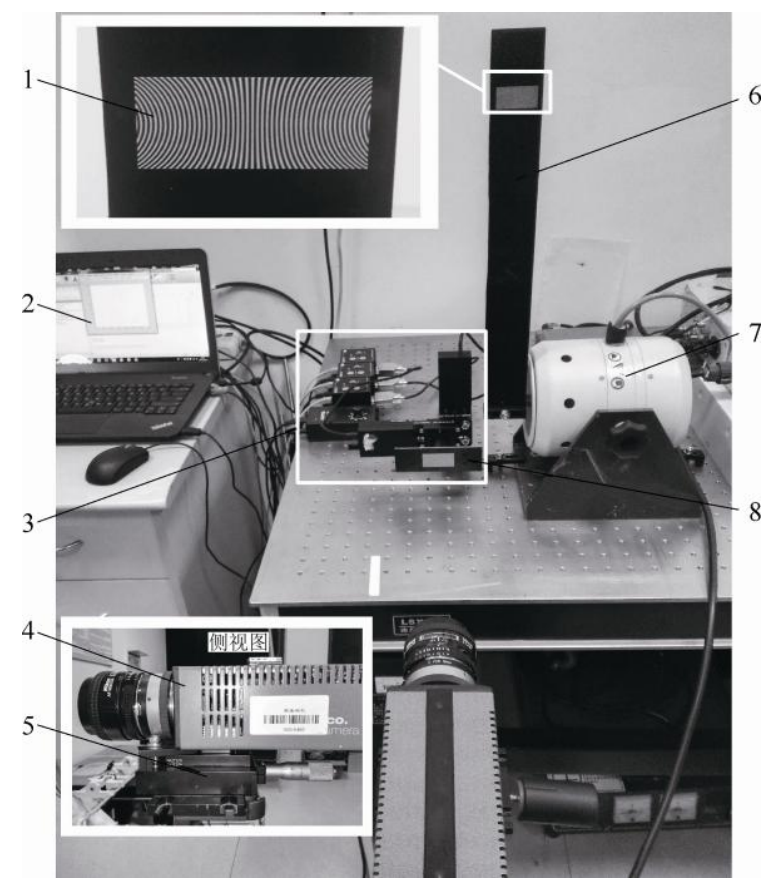

图 7 试验装置图

1. 位感条纹 2. 电脑 3. 三维移动平台 4. 高速相机 5 . 移动平台 6. 悬臂梁 7. 激振器 8 . 轻质平板

试验中首先对安装于三维电动移动平台的轻质 平板结构的已知空间运动进行测量。该三维电动移 动平台由三个一维电动移动平台 (THORLAB : MTS25/M-Z8)搭建而成, 并通过 Matlab 软件控制每 个方向一维电动移动平台的运动, 进而实现轻质平 板结构的三维空间运动。平板结构运动前在其表面 粘贴好位感条纹, 放置好高速相机使位感条纹成像 于图像传感器中心位置, 调整好成像镜头和图像传 感器的各参数使位感条纹图像清晰。试验中高速相 机的成像范围设置为 640 像素 $\times 330$ 像素, 曝光时间 设置为 $1 \mathrm{~ms}$, 并在两曝光时间点之间设置 $4 \mathrm{~ms}$ 的 延时, 在该参数设置条件下高速相机的采样帧率为 200 帧/s。在实际的测量过程中, 图像的对比度和成 像质量可能会受到外界光照条件的影响。如果测量 环境的光照条件不够理想可以使用外部光源照射位 感条纹，以提高其成像质量。

设置好位感条纹和三维测量系统后, 分别对 $X$ 、 $Y$ 和 $Z$ 三个方向的一维电动移动平台施加位移幅值 为 $1 \mathrm{~mm}$ 的往复运动, 其中各移动平台加速度和最 大速度都设置为 $1 \mathrm{~mm} / \mathrm{s}^{2}$ 和 $1 \mathrm{~mm} / \mathrm{s}$ 。经三维测量系 统测量得到的平板三维空间运动曲线如图 8a 所示, 其在 $X Y 、 X Z$ 和 $Y Z$ 投影平面的投影曲线可以更好地 观察其在各投影平面的运动情况。图 $8 \mathrm{~b}$ 为平板结构 在 $X 、 Y$ 和 $Z$ 方向上的时域运动曲线, 其往复运动 幅值与各移动平台设置的幅值相等。验证了该振动
测量方法各方向位移计算算法的正确性与准确性, 也说明了该基于单个位感条纹的结构三维振动测量 系统可实现结构三维振动的同步测量。

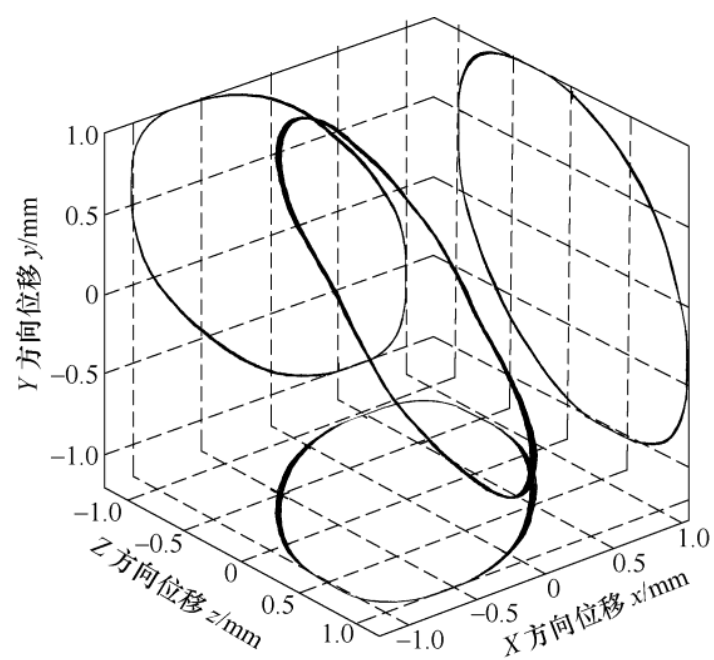

(a) 三维移动平台上平板结构的空间运动曲线

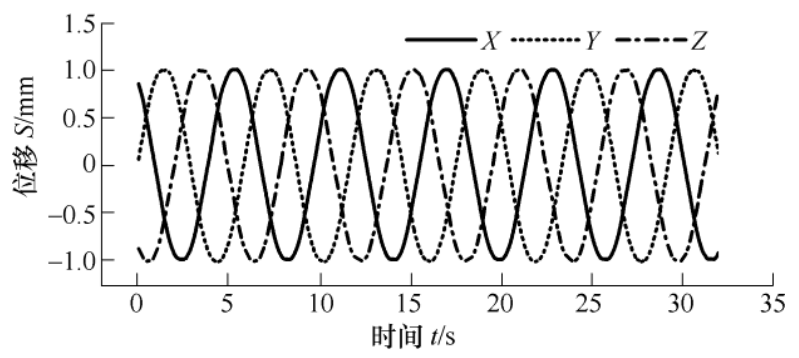

(b) 三维移动平台上平板结构不同方向的运动曲线

图 8 平板结构三维位移测量结果

为了对该测量系统的测量精度进行验证, 采用 三维电动移动平台分别在 $X 、 Y$ 和 $Z$ 三个方向产生 5 步位移增量为 $10 \mu \mathrm{m}$ 的阶梯运动。在运动过程中采 用提出的动态测量系统对该位移进行测量, 并通过 提出的位移提取算法得到结构三个方向的阶梯位移 曲线如图 9 所示。由图可知, 系统可以分辨三个方 向步距为 $10 \mu \mathrm{m}$ 的阶梯运动。但是由于本系统中相 机单个像素点尺寸和位深分别为 $12 \mu \mathrm{m} \times 12 \mu \mathrm{m}$ 和 8 位, 采集得到的位感条纹信噪比较差, 导致计算条 纹密度信息时的误差较大。所以系统 $X$ 方向的位移 曲线噪声水平较大。但是系统 $X$ 和 $Y$ 方向的位移信 号信噪比较高, 可实现微米量级位移的分辨与测量。

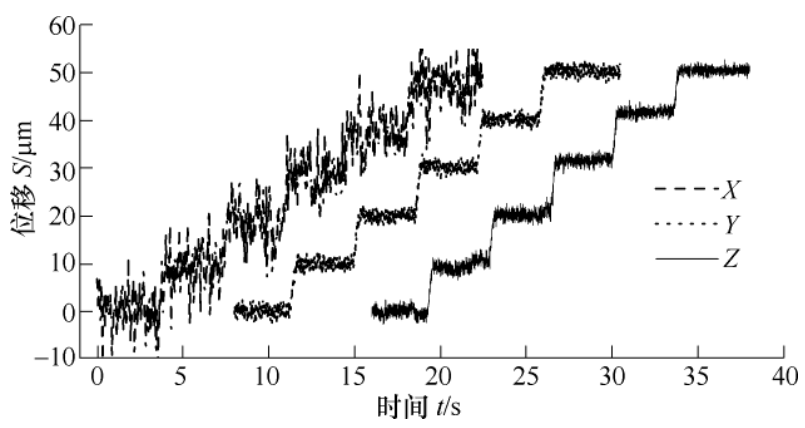

图 9 结构三维阶梯位移试验测量结果 
为进一步验证该三维振动测量系统对大位移结 构的振动测量性能, 采用该测量系统对悬臂梁结构 的三维振动进行测量。因为悬臂梁在摆动时只在一 个平面内运动, 为了增加另一个维度的振动, 将该 悬臂梁固定于激振器的输出端, 如图 7 所示。激振 器输出端的振动信号幅值和频率可以由信号发生器 (Agilent 33220)输出信号的幅值和频率控制。试验 前, 将位感条纹粘贴于悬臂梁自由端附近, 并放置 调整好高速相机。然后在悬臂梁自由端施加力矩后 释放, 使其自由摆动。同时, 激振器输出端输出 $1 \mathrm{~Hz}$ 的振动信号, 使悬臂梁整体横向振动。

系统对该振动悬臂梁的测量结果如图 10 所示。 其中图 10a 为悬臂梁在 $X$ 和 $Y$ 方向的时域空间摆动 曲线, 可以看出悬臂梁结构的摆动幅度逐渐衰减。 该空间曲线左侧的投影曲线可以观察悬臂梁在 $X Y$ 平面的摆动轨迹; 空间曲线下方的投影为悬臂梁在 $X$ 方向时域位移曲线, 由该衰减曲线的幅值变化可 以看出, 该测量系统在 $X$ 方向可实现从微米量级到 厘米量级的振动测量; 空间曲线后侧的投影曲线为 该悬臂梁在 $Y$ 方向的时域位移曲线。图 $10 \mathrm{~b}$ 为悬臂 梁结构在 $Z$ 方向的时域振动曲线, 通过能量重心频 谱校正法校正后得到该单频振动信号的振动频率为 $1.0001 \mathrm{~Hz}$, 与输入激振器信号的频率几乎相等。进

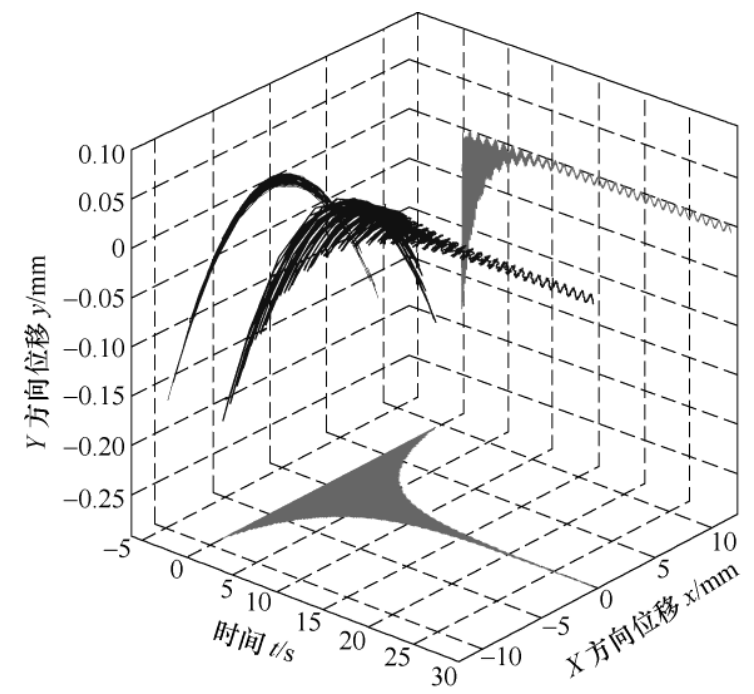

(a) $X$ 和 $Y$ 方向测量的时域空间振动曲线及其投影

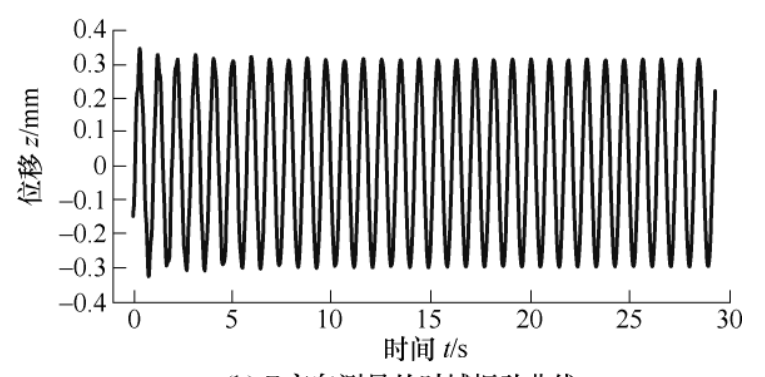

(b) $Z$ 方向测量的时域振动曲线

图 10 悬臂梁结构三维振动测量结果
一步验证了基于位感条纹的三维振动测量系统的可 行性与准确性。

该测量系统采用的高速相机在全分辨率条件下 (1 280×1 024 像素)最大帧率为 636 帧 $/ \mathrm{s}$ 。根据采样 定律, 以采样频率为振动信号最高频率的 2.5 倍计 算, 该相机在全分辨率下可测量的理论最大振动频 率约为 $255 \mathrm{~Hz}$, 因此可以满足实际工程中一些大型 工程结构大振幅、低频率振动的三维振动测量。但 是该高速相机图像传感器的成像范围可以根据成像 位感条纹的实际图像大小进行设置, 在低成像范围 条件下可实现几 $\mathrm{kHz}$ 的高帧率采集, 也适用于结构 高频振动的测量。为了验证该测量系统对高频三维 振动位移的测量效果, 采用该测量系统对扫频激励 下的悬臂梁三维振动进行测量。试验中采用螺钉将 悬臂梁一端固定于激振器的输出端, 然后将位感条 纹粘贴于距悬臂梁固定端 $10 \mathrm{~cm}$ 的正面位置。为了对 该系统的测量效果进行验证, 试验中也采用基于三 维加速度传感器的动态测试系统进行同步测量。试 验中, 将三维加速度传感器( $\mathrm{PCB}$, 型号: 356B21) 安装于距悬臂梁固定端 $10 \mathrm{~cm}$ 的背面位置, 并采用动 态测试采集系统(东华测试, DH5922)对加速度信号 进行同步采集。激振器输出扫描频率范围为 $0 \sim 300$ $\mathrm{Hz}$, 扫描周期为 $6 \mathrm{~s}$ 激励信号。高速相机的成像范围 设置为 600 像素 $\times 244$ 像素, 曝光时间设置为 $0.5 \mathrm{ms,}$ 该设置下高速相机的采样频率为 $2 \mathrm{kHz}$ 。扫频激励下 悬臂梁结构的三维振动位移测量结果如图 11 所示。

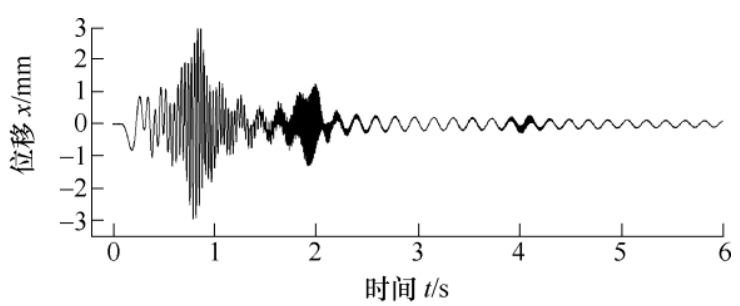

(a) $X$ 方向时域振动曲线

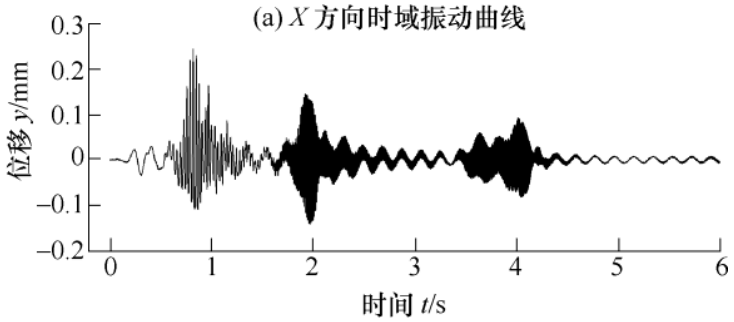

(b) $Y$ 方向时域振动曲线

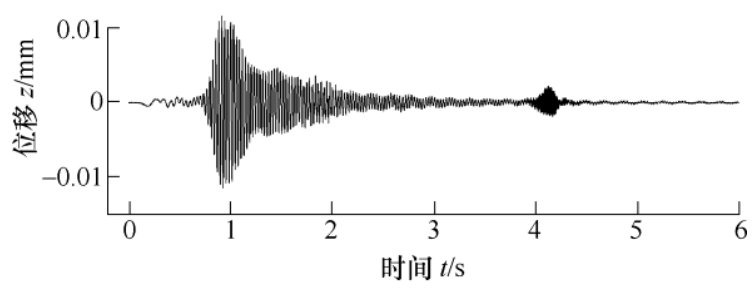

(c) $Z$ 方向时域振动曲线

图 11 扫频激励下悬臂梁结构三维振动位移测量结果 
图 11a、11b 和 $11 \mathrm{c}$ 分别为结构在 $X 、 Y$ 和 $Z$ 方 向上的时域振动位移曲线。由图可知, 当扫频激励 信号的频率与梁结构的共振频率相近时梁结构将发 生共振, 其振动幅值将变大。因为激振器沿梁的 $X$ 方向进行激励, 所以沿 $X$ 方向的振幅较大, 其最大 振动幅值约为 $3 \mathrm{~mm}$ 。而沿 $Y$ 和 $Z$ 方向上的振动幅 值较小, 最大振动幅值分别为 $230 \mu \mathrm{m}$ 和 $12 \mu \mathrm{m}$ 。 因此, 该测量系统可以实现高频振动结构三维位移 的测量。

因为三维加速度传感器测量得到的是悬臂梁的 三维加速度信号, 在时域上与三维位移信号难以对 比。因此通过悬臂梁动态信号的频域信号对比分析 对测量系统的测量性能进行对比验证, 对比结果如 图 12 所示。图 12a、12b 和 $12 \mathrm{c}$ 分别为三维加速度 传感器和位感条纹传感器在 $X 、 Y$ 和 $Z$ 方向上振动 加速度信号和振动位移信号的频谱对比结果。由图 可知, 三维加速度传感器和位感条纹都可以得到悬 臂梁结构的各阶共振频率。因此, 该测量系统可用 于一些高频振动结构三维振动的同步测量。

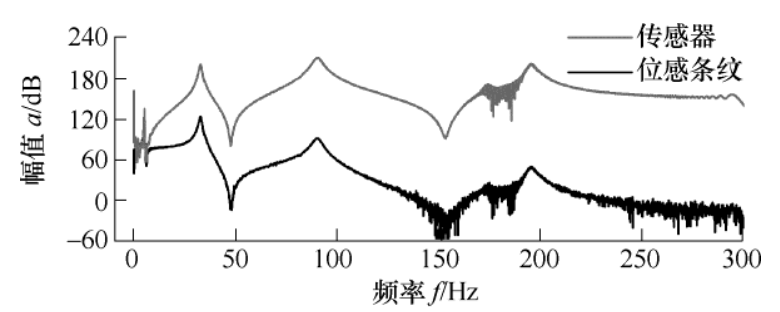

(a) $X$ 方向频域曲线对比

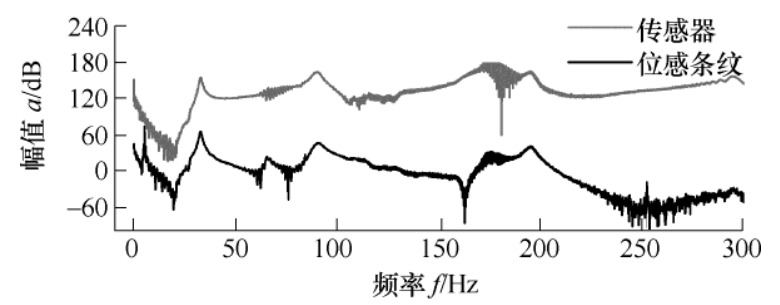

(b) $Y$ 方向频域曲线对比

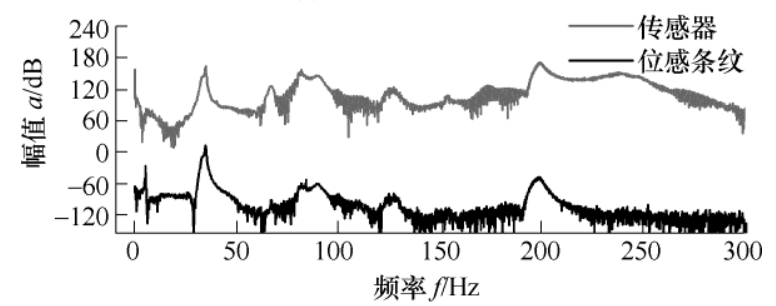

(c) $Z$ 方向频域曲线对比

图 12 加速度传感器和位感条纹测量动态信号的频谱对比

本文提出的位感条纹三维振动位移测量系统, 只需将设计的位感条纹粘贴于或者喷涂于待测结构 的平整表面, 采用高速相机采集随结构振动的位感 条纹图, 并运用提出的三维位移算法对采集的位感 条纹图像进行处理即可实现结构三维振动位移的同 步测量。并且轻质位感条纹的质量较小, 对于一般
结构其质量几乎可以忽略不计, 不会对测量结构引 入附加质量而影响其振动特性。特别适用于一些轻 质、大柔度或宽振幅范围振动结构的测量。因此, 可用于结构动态特征参数的准确测量与评估, 实现 结构的状态监测或缺陷检测, 从而保证工程结构的 安全性及其可靠性。

\section{4 结论}

(1) 提出一种结构三维振动同步测量的新方 法, 该方法采用新型位感条纹作为结构三维振动传 感器, 采用高速相机作为探测器实现结构的三维振 动同步测量。

(2) 分析影响该测量系统测量精度的各种影响 因素, 模拟结果表明, 该测量系统 $X 、 Y$ 和 $Z$ 三个方 向的测量精度各不相同, 但是它们之间的精度差别 随着位感条纹图像信噪比提高而减小; 并且它们的 测量精度都随着位感条纹图像分辨率和信噪比的增 高而提高。

(3) 搭建试验测量系统并对安装于三维电动移动 平台平板结构和悬臂梁结构的三维振动进行测量, 试 验结果表明, 该基于位感条纹和高速相机的三维振动 同步测量系统可精确地实现结构微米量级到厘米量 级大振幅范围内三维振动的同步测量。

\section{参 考 文 献}

[1] 徐超, 张一凡, 韩晓明, 等. 基于机器视觉的大柔性结 构振动位移测量 $[\mathrm{J}]$. 振动测试与诊断, 2017, 37(4): 781-786, 846.

XU Chao, ZHANG Yifan, HAN Xiaoming, et al. Vibration displacement measurement of large flexible structures based on machine vision[J]. Journal of Vibration, Measurement \& Diagnosis, 2017, 37(4): 781786,846 .

[2] FU Yu. Low-frequency vibration measurement by temporal analysis of projected fringe patterns[J]. Optics and Lasers in Engineering, 2010，48(2): 226-234.

[3] TAY C J. New method for measuring dynamic response of small components by fringe projection[J]. Optical Engineering, 2003, 42(6): 1715.

[4] MITCHELL A. Optical modal analysis using white-light projected fringes[J]. Experimental Mechanics, 2005, 45(3): 250-258.

[5] 陈露, 王效惠, 李雪, 等. 基于条纹投影的振动扬声器 形变测量 $[J]$. 光学与光电技术, $2015,13(1)$ : 22-27.

CHEN Lu, WANG Xiaohui, LI Xue, et al. Deformation 
measurement of a vibrating speaker based on fringe projection[J]. Optics \& Optoelectronic Technology, 2015, 13(1): $22-27$.

[6] FUKUDA Y, FENG M Q, NARITA Y, et al. Vision-based displacement sensor for monitoring dynamic response using robust object search algorithm[J]. Sensors Journal, IEEE, 2013， 13(12): 4725-4732.

[7] BUSCA G, CIGADA A, MAZZOLENI P, et al. Vibration monitoring of multiple bridge points by means of a unique vision-based measuring system[J]. Experimental Mechanics, 2014, 54(2): 255-271.

[8] 陈苏, 陈国兴, 韩晓健, 等. 基于计算机视觉的位移测 试方法研究与实现 $[\mathrm{J}]$. 振动与冲击, 2015, 34(18): 73-78, 99.

CHEN Su, CHEN Guoxing, HAN Xiaojian, et al. Development of vision-based displacement test method[J]. Journal of Vibration and Shock, 2015, 34(18): 73-78, 99.

[9] DOIGNON C, KNITTEL D, MAURICE X. A visionbased technique for edge displacement and vibration estimations of a moving flexible web[J]. Instrumentation and Measurement, IEEE Transactions on, 2008，57(8): 1605-1613.

[10] FENG M, FUKUDA Y, FENG D, et al. Nontarget Vision sensor for remote measurement of bridge dynamic Response[J]. Journal of Bridge Engineering, 2015，20: 1-12.

[11] KHUC T, CATBAS F N. Completely contactless structural health monitoring of real-life structures using cameras and computer vision[J]. Structural Control and Health Monitoring, 2017, 24(1): 1-17.

[12] 季云峰. 无目标计算机视觉技术在斜拉索振动测试中 的应用研究 $[\mathrm{J}]$. 振动与冲击, 2013，32(20): 184-188, 202.

JI Yunfeng. Application of non-target computer-visionbased technique in stay-cable vibration measurement [J]. Journal of Vibration and Shock, 2013, 32(20): 184-188, 202.

[13] PAN Bing, QIAN Kemao, XIE Huimin, et al. Twodimensional digital image correlation for in-plane displacement and strain measurement : A review[J]. Measurement Science and Technology, 2009, 20(6): $1-17$.

[14] FAZZINI M, MISTOU S, DALVERNY O, et al. Study of image characteristics on digital image correlation error assessment[J]. Optics and Lasers in Engineering, 2010, 48(3): 335-339.

[15] YUAN Yuan, HUANG Jianyong, FANG Jing, et al. A self-adaptive sampling digital image correlation algorithm for accurate displacement measurement[J]. Optics and Lasers in Engineering, 2015，65(1): 57-63.

[16] HELFRICK M N, NIEZRECKI C, AVITABILE P, et al. 3D digital image correlation methods for full-field vibration measurement[J]. Mechanical Systems and Signal Processing, 2011, 25(3): 917-927.

[17] 陈忠, 陈教豆. 基于双目立体视觉与数字散斑图像相关 的全场振动测量 $[\mathrm{J}]$. 振动与冲击, 2015, 34(13) : 121-126.

CHEN Zhong, CHEN Jiaodou. Full-field vibration measurement based on binocular stereo vision and digital speckle image correlation[J]. Journal of Vibration and Shock, 2015, 34(13): 121-126.

[18] 歹英杰, 汪伟, 邓士杰, 等. 基于立体机器视觉的动目 标空间位姿测试研究 [J]. 振动与冲击, 2015, 34(16): 188-194, 212.

DAI Yingjie, WANG Wei, DENG Shijie, et al. Position and posture measurement of dynamic target based on stereo machine vision[J]. Journal of Vibration and Shock, 2015, 34(16): 188-194, 212.

[19] ZHONG Shuncong, SHEN Hao, SHEN Yaochun. Real-time monitoring of structural vibration using spectral-domain optical coherence tomography[J]. Optics and Lasers in Engineering, 2011，49(1): 127-131.

[20] ZHONG Jianfeng, ZHONG Shuncong, ZHANG Qiukun. Two-dimensional optical coherence vibration tomography for low-frequency vibration measurement and responseonly modal analysis[J]. Mechanical Systems and Signal Processing, 2016, 79: 65-71.

[21] ZHONG Jianfeng, ZHONG Shuncong, ZHANG Qiukun, et al. Vision-based measurement system for structural vibration monitoring using non-projection quasiinterferogram fringe density enhanced by spectrum correction method[J]. Measurement Science and Technology, 2017, 28(1): 1-12.

[22] ZHONG Jianfeng, ZHONG Shuncong, ZHANG Qiukun. Quasi-OCVT technique for response-only experimental modal analysis of beam-like structures[J]. Structural Control and Health Monitoring, 2017, 24(11): 1-10.

[23] ZHONG Shuncong, ZHONG Jianfeng, ZHANG Qiukun, et al. Quasi-optical coherence vibration tomography technique for damage detection in beam-like structures based on auxiliary mass induced frequency shift[J]. Mechanical Systems and Signal Processing, 2017，93: 241-254.

[24] 谢明, 丁康. 频谱分析的校正方法 [J]. 振动工程学报, 1994, 7(2): 172-180.

XIE Ming, DING Kang. Correction method for spectrum 
analysis[J]. Journal of Vibration Engineering, 1994, 7(2): 172-180.

作者简介: 钟剑锋, 男, 1988 年出生, 博士, 讲师。主要研究方向为机 电系统状态监测与智能诊断技术研究。

E-mail: zhongjianfeng@fzu.edu.cn
钟舜聪(通信作者), 男, 1976 年出生, 博士, 教授, 博士研究生导师。 主要研究方向为结构健康监测和设备故障诊断、无损检测和定量评价、 光学精密诊断仪器和太赫兹技术等。

E-mail: sczhong@fzu.edu.cn

彭志科, 男, 1974 年出生, 博士, 教授, 博士研究生导师。主要研究方 向为非线性振动理论及方法、振动信号分析、设备故障诊断等。

E-mail: z.peng@sjtu.edu.cn 